\title{
Diagnostic strategies for early pancreatic cancer
}

\author{
Keiji Hanada Akihito Okazaki $\cdot$ Naomichi Hirano Yoshihiro Izumi · \\ Yuji Teraoka $\cdot$ Juri Ikemoto $\cdot$ Kozue Kanemitsu $\cdot$ Fumiaki Hino • \\ Toshikatsu Fukuda $\cdot$ Shuji Yonehara
}

Received: 25 November 2014 / Accepted: 1 December 2014/Published online: 14 December 2014

(C) Springer Japan 2014

\begin{abstract}
Diagnosis of pancreatic cancer (PC) at an early stage with curative surgery is the approach with the potential to significantly improve long-term patient outcome. Recently, some reports showed that patients with pancreatic tumors smaller than $10 \mathrm{~mm}$ showed a favorable prognosis. However, the rate of tumor detection on computed tomography in patients with small pancreatic tumors is low. For the diagnoses of PC with tumors smaller than $10 \mathrm{~mm}$, the rate of tumor detection was higher on endoscopic ultrasonography (EUS) than on computed tomography or other modalities, and histologic diagnosis using EUS-guided fine-needle aspiration was helpful in confirming the diagnosis. For the diagnosis of PC in situ, EUS and magnetic resonance cholangiopancreatography may play important roles in detecting the local irregular stenosis of the pancreatic duct. Endoscopic retrograde pancreatography and sequential cytodiagnosis using pancreatic juice obtained by endoscopic nasopancreatic drainage multiple times was useful in the final diagnosis of PC in situ. At
\end{abstract}

Part of this review was presented at The 4th International Forum of the 100th General Meeting of the Japanese Society of Gastroenterology.

K. Hanada $(\bowtie) \cdot$ A. Okazaki · N. Hirano · Y. Izumi ·

Y. Teraoka $\cdot$ J. Ikemoto $\cdot$ K. Kanemitsu $\cdot$ F. Hino

Department of Gastroenterology, Onomichi General Hospital,

1-10-23 Hirahara, Onomichi 722-8508, Japan

e-mail: kh-ajpbd@nifty.com

T. Fukuda

Department of Surgery, Onomichi General Hospital,

1-10-23 Hirahara, Onomichi 722-8508, Japan

S. Yonehara

Department of Pathology, Onomichi General Hospital,

1-10-23 Hirahara, Onomichi 722-8508, Japan present, improving survival lies in identifying those individuals with high-risk factors or precursor lesions through an effective screening method. For example, these should include ultrasonography, various biological markers, or national familial pancreatic cancer registration. Additionally, the relationship between specialists in PC from medical centers and practicing physicians plays an important role in the early diagnosis of PC.

Keywords Early diagnosis - Endoscopic nasopancreatic drainage Carcinoma in situ of pancreatic cancer . Physician community

\section{Introduction}

Patients with pancreatic cancer (PC) have an extremely poor prognosis, given that in many of them $\mathrm{PC}$ is diagnosed at an advanced stage. In Japan, more than 26,000 patients died of PC in 2011 [1]. This poor prognosis is attributed to the difficulty in diagnosing PC early [2,3]. It has been suggested that many cases of PC arise from the branch ducts [4]. The diameter of the main pancreatic duct (MPD) is about 2 or $3 \mathrm{~mm}$ in an adult. If small duct cell pancreatic carcinoma is present in the branch of the pancreatic duct, the small cystic lesion or the dilatation of the duct would be detected because of the obstruction.

According to the recent Japan Pancreatic Cancer Registry (JPCR), the 5-year survival rate of patients with tumors smaller than $10 \mathrm{~mm}$ reached $80.4 \%$ [5]. If the tumor is $10 \mathrm{~mm}$ or smaller, most of the cases are Union for International Cancer Control (UICC) stage Ia, with a favorable 5-year survival (68.7\%). However, only a few patients with tumors smaller than $10 \mathrm{~mm}$ have been registered in the JPCR. 
In this article, we present a review of the current topics and problems regarding the early diagnosis of PC.

\section{Risk factors for PC}

In Japan, the clinical guidelines for PC based on evidencebased medicine were published by the Japan Pancreas Society (JPS) in 2006 and were revised to the third version in 2013 [6]. The guidelines show an algorithm for the diagnosis and treatment of PC. In the session of clinical question 1, some risk factors were suggested for the development of PC (Table 1). There are a few reports on the development of PC during the follow-up of patients with branch duct intraductal papillary mucinous neoplasms (IPMNs) [7, 8] or pancreatic cysts [9]. Uehara et al. [7] reported that the 5-year rate of PC development was $6.9 \%$ during follow-up of patients with branch duct IPMNs. Tada et al. [9] reported that patients with pancreatic cystic lesions were at a high risk of $\mathrm{PC}$, with a standardized incidence rate of $22.5 \%$. A multicenter study from a working group of the JPS recently reported that PC distinct from branch duct IPMNs developed in seven $(2.0 \%)$ of 349 patients during the follow-up period (median 3.7 years) [10]. From these reports, the guidelines recommended that IPMNs and pancreatic cysts should be carefully followed as premalignant diseases of PC [6]. Additionally, another JPS working group reported that PC concomitant with IPMN may have more favorable biological behaviors or may be diagnosed earlier than ordinary PC [11]. These reports suggest that the regular follow-up of IPMN patients may play an important role in the early diagnosis of PC.

\section{Surveillance of high-risk groups}

With regard to family history, the National Familial Pancreas Tumor Registry at Johns Hopkins Hospital reported

Table 1 Risk factors for pancreatic cancer

Family history
Pancreatic cancer
Hereditary pancreatic cancer syndrome
Accompanying diseases
Diabetes mellitus
Obesity
Chronic pancreatitis
Hereditary pancreatitis
Intraductal papillary mucinous neoplasm
Pancreatic cysts
Habits
Tobacco use
Heavy drinking

that the risk of $\mathrm{PC}$ was 6.79-fold higher in the relatives of patients with familial PC, 2.41-fold higher in relatives of patients with sporadic PC, and 2.14-fold higher in spouses of patients with PC [12]. Canto et al. [13] have reported that endoscopic ultrasonography (EUS)-based screening of asymptomatic high-risk individuals (familial PC relatives) can detect prevalent resectable cases of PC. Tumor syndromes associated with an increased risk of PC include familial atypical multiple mole melanoma, hereditary breast cancer (BRCA2), Lynch syndrome, p16 Leiden mutation, and Peutz-Jeghers syndrome. The risk of PC ranges from $5 \%$ in individuals with BRCA2 mutation to $36 \%$ in individuals with Peutz-Jeghers syndrome [14-16]. However, the optimal strategy for surveillance in high-risk groups has not been determined. Poley et al. [17] observed asymptomatic PC in three patients $(7 \%)$, including one patient with stage I PC, and IPMN-like lesions in six patients $(16 \%)$ using EUS in a heterogeneous group of 44 individuals at high risk of PC. During surveillance of 73 p16 Leiden mutation carriers via magnetic resonance imaging (MRI) and magnetic resonance cholangiopancreatography (MRCP), with a median follow-up period of 4 years, precursor lesions were observed in nine patients and PC was observed in seven patients, including four patients with tumors smaller than $20 \mathrm{~mm}$ [18]. An effective screening strategy for the high-risk groups should be published. In Japan, JPS initiated the familial pancreas tumor registry for the early diagnosis of PC in July 2013.

\section{Screening strategies}

\section{Biomarkers}

With regard to blood-based biomarkers, carbohydrate antigen 19-9 (CA19-9) remains the most commonly used tumor biomarker for following the therapeutic outcomes of PC. However, there are several concerns with its use. Only $50 \%$ of cases of PC with tumors smaller than $20 \mathrm{~mm}$ are associated with a rise in CA19-9 levels [19]. In addition, its levels are also increased in other gastrointestinal malignancies and benign pancreatic diseases. Other serum tumor markers such as carcinoembryonic antigen, Duke pancreatic monoclonal antigen type 2 (DUPAN-2), elastase 1, and SPan-1 could also be used, but with the same caveat [20]. Many putative markers have failed to meet their initial promise for the early diagnosis of PC. Attempts are being made to identify a panel of biomarkers based on genetic, immunological, and biochemical changes in PC.

Human serum metabolomics using gas chromatography-mass spectrometry has been reported to be useful for discriminating patients with PC from healthy volunteers [21]. Kobayashi et al. [22] developed a serum 
metabolomics-based diagnostic model for PC using multiple logistic regression analysis. This model possessed higher accuracy than conventional tumor markers especially in diagnosing resectable PC. It is a promising method for improving the prognosis of PC via its early diagnosis and accurate discrimination from chronic pancreatitis. Recent research has revealed that microRNAs (miRNAs) play an important role in the initiation, progression, and metastasis of cancer. As for the early diagnosis of $\mathrm{PC}, \mathrm{Yu}$ et al. [23] detected altered expression in 35 of 700 miRNAs in PanIN-3 lesions using quantitative real-time PCR. Further studies are warranted to profile the relationship between miRNAs and the stage of PC for their clinical use as biomarkers. Profiling miRNA in serum or pancreatic juice collected by endoscopic retrograde cholangiopancreatography (ERCP) may be regarded as the best prospect for miRNA-based biomarker development [23, 24].

As for the endoscopic approach, Mori et al. [25] established a minimally invasive and simple screening test for early-stage PC using the duodenal juice. After the sideview endoscope had been positioned in the second portion of the duodenum, duodenal juice was collected for $5 \mathrm{~min}$ by gentle pushing of the duodenal mucosa using an ERCP cannula. The sensitivity of CEA and that of S100P, a small protein composed of 95 amino acids, in duodenal juice to diagnose PC were higher than the sensitivities of serum tumor markers or duodenal juice cytology. More studies should focus on identifying the altered genetic and molecular expression profiles of various proteins for the diagnosis of PC at an early stage.

\section{Screening programs for high-risk individuals}

Recently, there have been multiple programs developed for the screening of PC in high-risk individuals in a research setting. The largest program is Cancer of the Pancreas Screening (CAPS) led by Johns Hopkins University [13]. In the CAPS 1 study, 36 patients were screened using only EUS. Six masses were found, two of which had malignant and premalignant lesions. The diagnostic yield of screening was $5.3 \%$, and all patients were alive after surgical therapy for more than 5 years [13]. In the CAPS 2 study, screening was performed prospectively using annual EUS and computed tomography (CT). If there was an abnormal finding, ERCP was offered. Seventy-eight high-risk patients and 149 controls were examined. The diagnostic yield of screening was $10 \%$. Eight patients had confirmed pancreatic neoplasms as determined after surgery and using EUS-guided fine-needle aspiration (EUS-FNA) [26]. In the CAPS 3 study, researchers screened 216 adult asymptomatic high-risk individuals for $\mathrm{PC}$ at five American academic medical centers using CT, MRI, and EUS. Ninety-two of these individuals had at least one pancreatic mass or a dilated pancreatic duct. CT, MRI, and EUS revealed abnormal pancreatic findings in 11,33 , and $43 \%$ of highrisk individuals, respectively. These abnormal findings were proven or suspected neoplasms in 85 patients. Among these patients, three had high-grade dysplasia in IPMNs or multiple intraepithelial neoplasms [27]. These results suggested that screening of asymptomatic high-risk individuals for PC may frequently reveal small cystic lesions, including curable noninvasive neoplasms, and that EUS and MRI may be better than CT for the early diagnosis of pancreatic neoplasms [28]. Kamata et al. [29] reported the value of EUS in early diagnosis of PC in patients with IPMN. The 102 patients whose branch duct IPMNs lacked mural nodules/symptoms and thus did not qualify for resection were followed up using semiannual EUS and annual ultrasonography, CT, and MRI. Eleven IPMNconcomitant PCs were diagnosed at the first examination. Additionally, seven IPMN-concomitant PCs (whose tumor sizes ranged from 7 to $30 \mathrm{~mm}$ ) were detected during follow-up. The 5-year survival rates for IPMN-concomitant PC development were $8.8 \%$. Throughout the study period, including at follow-up, EUS was significantly better at detecting IPMN-concomitant PCs than other modalities. Further studies and evidence are warranted to clarify how to treat patients with abnormal findings.

\section{Imaging of $\mathrm{PC}$ with tumors smaller than $10 \mathrm{~mm}$}

Surprising advances were reported in many imaging modalities, including ultrasonography, EUS, CT, MRI, endoscopic retrograde pancreatography (ERP), and ${ }_{18} \mathrm{~F}-$ fluorodeoxyglucose positron emission tomography (FDGPET) in the previous 10 years. Imaging is involved in all aspects of clinical management of PC, including diagnosis and characterization of the tumor. However, these modalities often fail to detect tumors smaller than $10 \mathrm{~mm}$. The sensitivity and accuracy of the diagnosis of small pancreatic tumors has been improved significantly; however, it is still difficult to diagnose $\mathrm{PC}$ at an early stage.

Ultrasonography is a noninvasive imaging modality with a high resolution even without the use of any contrast media. Tanaka et al. [30] reported that a slight dilatation of the MPD (2.5 $\mathrm{mm}$ or more) and pancreatic cysts (5 $\mathrm{mm}$ or more) observed on ultrasonography were very important predictive signs of PC. They diagnosed PC in 12 patients (including three patients with stage 0 disease and two patients with stage IA disease) among 1,058 prospective follow-up patients (mean follow-up 75.5 months) and also recommended periodic checkups at least once a year for individuals with slight dilatation of the MPD or pancreatic simple cysts. However, ultrasonography may have some limitations in the early diagnosis of PC. The entire 
pancreas is difficult to visualize clearly on ultrasonography, and small tumors located in the head or tail are especially difficult to visulaize.

For multidetector row CT for the diagnosis of tumors smaller than $10 \mathrm{~mm}$, the sensitivity ranged from 33 to $44 \%$ [31, 32]. Takeshita et al. [33] reported CT findings in eight patients (including two with tumors smaller than $10 \mathrm{~mm}$ ). Early PC appeared as low attenuation in the early phase and as high attenuation to isoattenuation during the pancreatic and delayed phases with regard to the tumor size. They also reported that associated pancreatitis occurred in six patients with tumors measuring $12 \mathrm{~mm}$ or more, and that loss of normal lobular texture was observed in four patients with a tumor measuring $14 \mathrm{~mm}$ or more. Additionally, focal stenosis or obstruction of the MPD with dilatation of the distal MPD observed on curved reformation imaging may be important in the early diagnosis of PC. Hijioka et al. [34] reported a patient with a small pancreatic tumor measuring $15 \mathrm{~mm} \times 10 \mathrm{~mm}$. The tumor was markedly enhanced in the late phase of the scans of dynamic CT and MRI. Fibrous tissues were sparsely distributed in the tumor, and there was an increase in the number of dilated veins at the margin. These results suggested that marked tumor enhancement in the late phase on CT or MRI may be a characteristic finding of PC at an early stage.

FDG-PET is a noninvasive functional imaging technique that exploits the difference in the biochemistry of normal and abnormal tissues [35]. There have been a few reports indicating the value of FDG-PET in the diagnosis of small pancreatic tumors. Previously, two studies reported that the sensitivity of FDG-PET for tumors smaller than $20 \mathrm{~mm}$ ranged from 81.3 to $100 \%[36,37]$. However, Matsumoto et al. [38] conducted a retrospective study on data collected from 232 consecutive patients with suspected PC from 2006 to 2012. FDG-PET detected only $50 \%$ of stage 0 and stage I tumors, suggesting that FDG-PET was not effective in diagnosing early-stage PC. Further studies are warranted to clarify the value of FDG-PET during the diagnosis of pancreatic tumors smaller than $10 \mathrm{~mm}$.

Among various imaging modalities, EUS has a higher imaging resolution that makes possible the diagnosis of small pancreatic tumors [39, 40]. Recently, many institutions have used EUS as their standard procedure because of its ability to detect tumors smaller than $10 \mathrm{~mm}$ [41, 42]. The sensitivity of detecting tumors on EUS in $25 \mathrm{PC}$ patients with tumors smaller than $10 \mathrm{~mm}$ collected from eight Japanese high-volume centers was $84 \%$ [31]. Sakamoto et al. [32] reported that the sensitivity of detecting tumors in six PC patients with tumors smaller than $10 \mathrm{~mm}$ was $100 \%$ for EUS, $17 \%$ for ultrasonography, and $33 \%$ for contrast-enhanced CT. Yasuda et al. [43] retrospectively examined data on 132 patients with risk factors for PC, such as increased levels of tumor markers and/or pancreatic enzymes, and they dilated the MPD without an identifiable pancreatic mass on CT. Pancreatic tumors smaller than $10 \mathrm{~mm}$ on EUS were subsequently diagnosed in three patients. These results suggested that EUS should be recommended for early diagnosis of pancreatic tumors smaller than $10 \mathrm{~mm}$ with the dilatation of the MPD detected using previous imaging tests. Recently, EUS-FNA has been popular for cytological or histological confirmation of PC. Uehara et al. [44] reported that the accuracy of EUS-FNA was $96 \%$ in 23 pancreatic masses smaller than $10 \mathrm{~mm}$. In six lesions, 22-gauge needles were used and, in 17 lesions, 25-gauge needles were used. Takagi et al. [45] also reported the high accuracy of EUS-FNA (93\%) in 14 pancreatic masses smaller than $10 \mathrm{~mm}$. EUS-FNA is considered to be useful in the confirmation of pancreatic tumors smaller than $10 \mathrm{~mm}$.

\section{Diagnosis of pancreatic carcinoma in situ}

According to the recent JPCR, the 5-year survival rate of patients with UICC stage Ia PC is $68.7 \%$, and that of patients with UICC stage $0 \mathrm{PC}$ is $85.8 \%$ [5]. The survival rate of patients with stage Ia PC has not been sufficiently compared with that of patients with cancers of other organs; therefore, PC needs to be diagnosed at an earlier stage. The possibility of diagnosing a carcinoma located in the mucosa of the pancreatic duct may greatly improve the prognosis of PC. It has been difficult to diagnose pancreatic carcinoma in situ (PCIS) without the presence of a formed mass using various imaging modalities. There were a few reports on using imaging modalities to diagnose PCIS. Localized stenosis, focal ductal branch dilatation, and distal dilatation of the MPD were frequently detected on EUS and MRCP [46, 47]. Seki et al. [48] evaluated the ERCP findings of seven patients with PCIS. Irregularity, noncontinuous narrowing, granular defects, and dilatation were frequently observed on ERCP. Ikeda et al. [49] reported the value of balloon spot pancreatography for the diagnosis of small pancreatic tumors and the possibility of diagnosing PCIS located at the branch ducts. As for pathological findings of PCIS using resected specimens, localized pancreatitis has been reported in the parenchyma around PCIS and atypical epithelium [50-52]. EUS may make possible the diagnosis of localized pancreatitis around PCIS as a slightly low echoic lesion [47]. Further studies are warranted to confirm this possibility. Recently, some cases of PCIS have been diagnosed by cytodiagnosis using pancreatic juice [53, 54]. Mikata et al. [55] investigated 60 patients with pancreatic disease (including two patients with PCIS) using endoscopic nasopancreatic drainage (ENPD) multiple times. A 5-Fr or 6-Fr ENPD tube was placed into the MPD for up to 3 days, and 

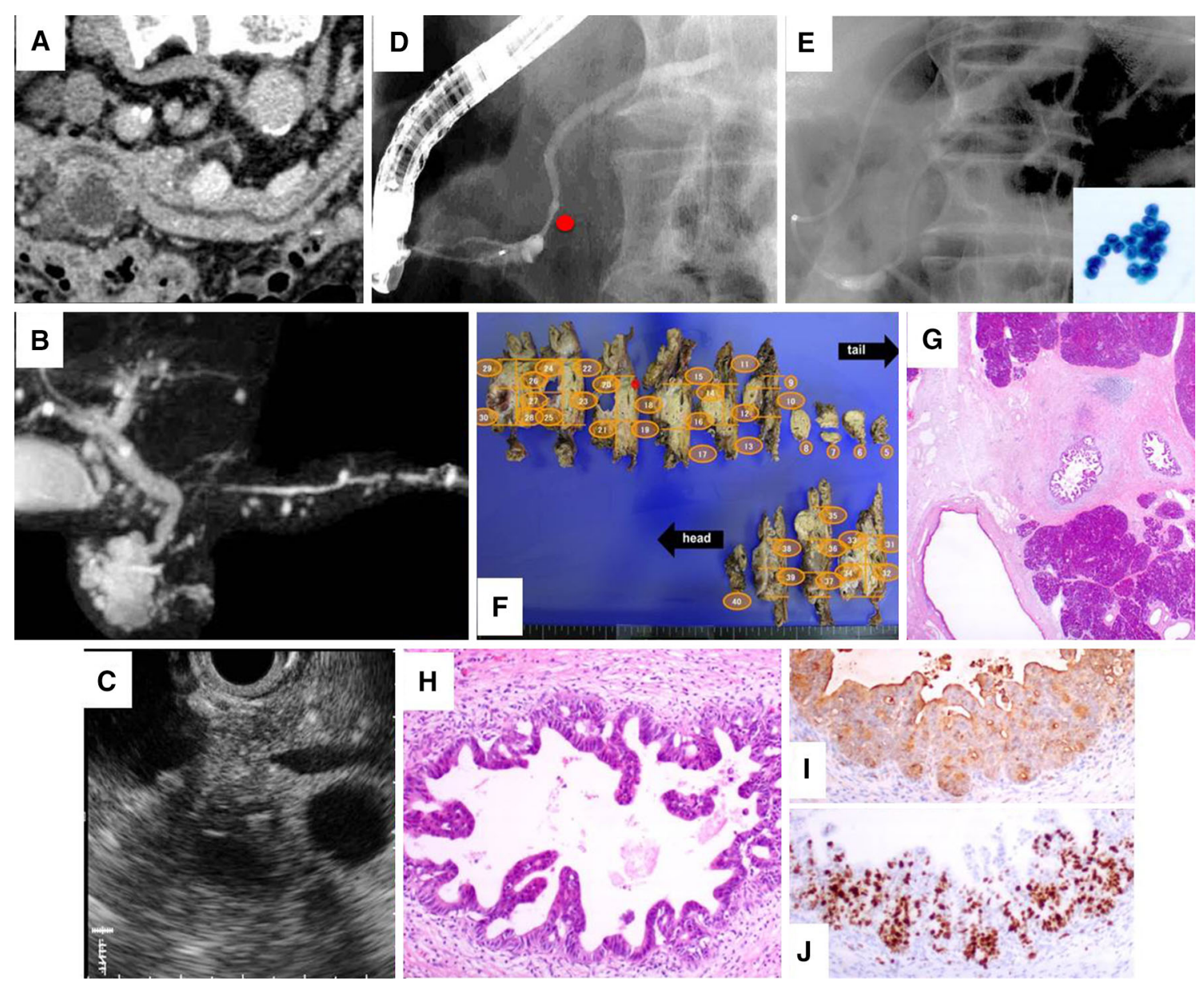

Fig. 1 An 82-year-old man with pancreatic carcinoma in situ (PCIS). Contrast-enhanced computed tomography (a) and magnetic resonance cholangiopancreatography (b) demonstrate irregular stenosis of the main pancreatic duct in the pancreatic body. Endoscopic ultrasonography indicated the stenosis with mild dilatation of the upstream duct (c). Endoscopic retrograde pancreatography revealed short irregular stenosis of the MPD in the pancreatic body (d), An endoscopic

cytological samples of pancreatic juice were collected up to six times in total. The sensitivity of the ENPD method for PCIS was $100 \%$ (two or two patients). Kimura et al. [56] investigated 157 patients with pancreatic disease (including three patients with PCIS) using ENPD multiple times. A 5-Fr ENPD tube was placed into the MPD for 1 day, and cytological samples of pancreatic juice were collected up to three times in total. The sensitivity of the ENPD method for PCIS was $100 \%$ (three of three patients). We investigated 20 patients presenting with focal stenosis and distal dilatation of the MPD (including seven patients with PCIS) using ENPD multiple times. A 5-Fr ENPD tube was placed for 1 day, and cytological samples were collected up to six times in total (Fig. 1). The results of ENPD cytodiagnosis and PC diagnosis showed a sensitivity of $100 \%$, a specificity of $83.3 \%$, and an accuracy of $95 \%$. In these 20 patients, no acute pancreatitis after ENPD tube placement nasopancreatic drainage tube was placed for 1 day, and cytological samples were collected six times, revealing adenocarcinoma (e). After distal pancreatectomy (f), pathological examination showed PCIS located at the irregular stenosis of the main pancreatic duct, with focal pancreatitis around PCIS (g, h). Carbohydrate antigen 19-9 (i) and p53 (j) tests were positive for PCIS. Red dots indicate the position of PCIS $(\mathbf{d}, \mathbf{f})$

occurred [51]. For the early diagnosis of PC with long-term survival, clinical guidelines for PC (2013) recommend the following: First, dilatation of the MPD and the presence of cysts are important indirect signs. MRCP and EUS are recommended, even when ultrasonography and CT fail to detect a mass directly. Second, when localized stenosis of the MPD is observed on the abovementioned imaging workup, ERCP with repeated cytology of the pancreatic juice is recommended [6].

\section{Relationship between specialists from medical centers and general practitioners for early diagnosis of PC}

The uneven distribution of specialists in pancreatic disease in Japan creates a disadvantage for rural and remote patients with PC, who may not receive a standard 


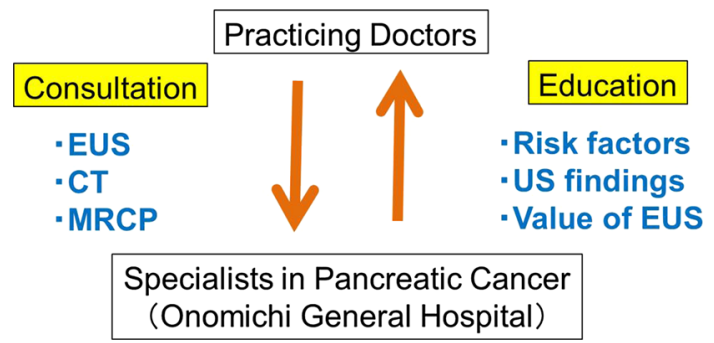

Fig. 2 The concept of the relationship between specialists in pancreatic cancer and general practitioners. $C T$ computed tomography, EUS endoscopic ultrasonography, MRCP magnetic resonance cholangiopancreatography, US ultrasonography

diagnosis. To resolve this problem, the relationship between specialists in PC (SPC) from medical centers and general practitioners is considered very important. Additionally, regional social programs should be established for the early diagnosis of PC in patients located in rural areas. Onomichi City is one of the rural cities located on the eastern side of Hiroshima prefecture in western Japan, and its total population is about 150,000 . Onomichi Medical Association has experienced difficulty in treating patients because of a lack of specialists in pancreatic disease despite an increasing number of patients with PC. In 2007, Onomichi Medical Association attempted to establish a social program for the early diagnosis of PC, with collaborations between SPC from medical centers and general practitioners (Onomichi Project) [57]. First, SPC from medical centers educated general practitioners about the risk factors of PC, abnormal ultrasonography findings, the importance of elevated serum levels of pancreatic enzymes, and the importance of repeated MRCP and EUS. Simultaneously, if general practitioners experienced a patient with the abovementioned problems, they aggressively consulted SPC from medical centers to check the entire lesion of the pancreas (Fig. 2). SPC first performed CT, MRCP, or EUS. If these imaging examinations revealed a mass in the pancreas, EUS-FNA was performed. If they revealed irregular stenosis in the MPD or revealed dilatation of the branch duct, ERCP was performed after informed consent had been obtained from the patient. If irregular stenosis of the MPD was observed on ERCP, the cytodiagnosis using pancreatic juice obtained by ENPD multiple times was performed (Fig. 3). From January 2007 to June 2013, 4,969 patients had consultations with SPC at our hospital. CT, MRI, and EUS were performed in 4,157, 2,303, and 1,692 patients, respectively. Among these, ERP was performed in 550 patients. ENPD and repeated cytology using pancreatic juice were performed in 59 patients. EUS-FNA was performed in 257 patients. As a result, adenocarcinoma was diagnosed in 338 patients. Surprisingly, of these patients, 13 had stage 0 PC (PCIS) and 26 had stage Ia or stage Ib PC (Fig. 4). For the detection of the early stages of PC, the

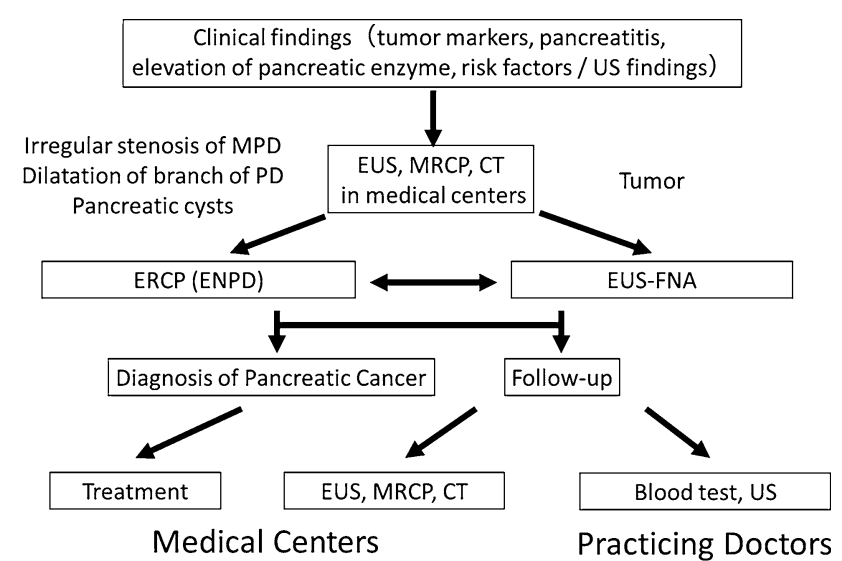

Fig. 3 The algorithm of the Onomichi Project for the early diagnosis of pancreatic cancer. $C T$ computed tomography, ENPD endoscopic nasopancreatic drainage, ERCP endoscopic retrograde cholangiopancreatography, EUS endoscopic ultrasonography, FNA fine-needle aspiration, $M P D$ main pancreatic duct, $M R C P$ magnetic resonance cholangiopancreatography, $P D$ pancreatic duct, $U S$ ultrasonography

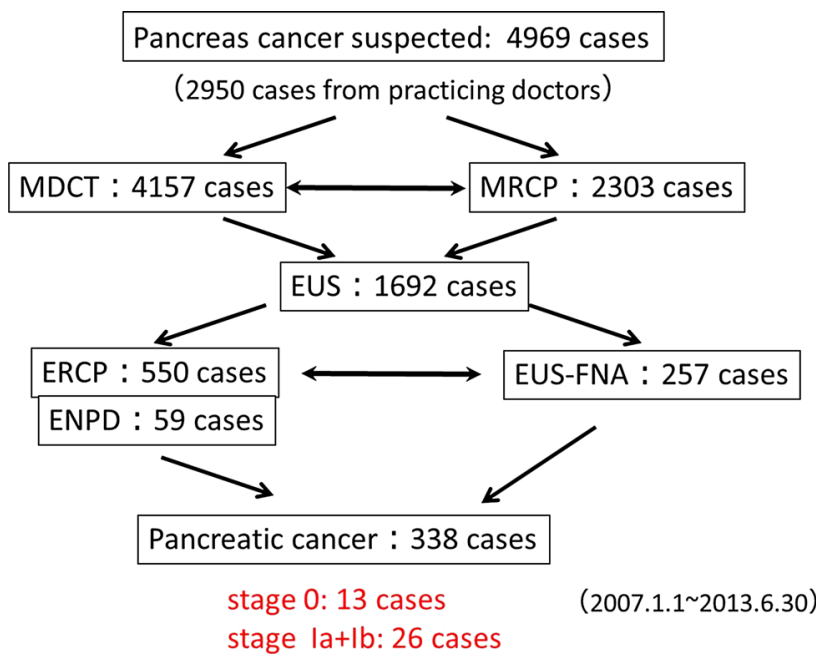

Fig. 4 The results of the Onomichi Project for the early diagnosis of pancreatic cancer. ENPD endoscopic nasopancreatic drainage, ERCP endoscopic retrograde cholangiopancreatography, EUS endoscopic ultrasonography, FNA fine-needle aspiration, $M D C T$ multidetector row computed tomography, $M R C P$ magnetic resonance cholangiopancreatography

relationship between SPC from medical centers and general practitioners in rural areas will play an important role in the future [57-59].

\section{Conclusions}

For the diagnosis of PC with tumors smaller than $10 \mathrm{~mm}$, the rate of tumor detection was higher with EUS than with CT or other modalities, and the histologic diagnosis using EUS-FNA was helpful in confirming the diagnosis. For the 
diagnosis of PCIS, EUS and MRCP play important roles in detecting local irregular stenosis of the pancreatic duct. ERP and sequential cytodiagnosis using pancreatic juice obtained by ENPD multiple times was useful in the final diagnosis of PCIS. Improving survival lies in identifying those individuals with high-risk factors or precursor lesions through an effective screening program. Effective screening programs should be established using methods involving ultrasonography, various biological markers, or national familial PC registration. Additionally, the relationship between SPC from medical centers and general practitioners plays an important role in the early diagnosis of PC.

Acknowledgment The authors are grateful to all members of Onomichi Medical Association for their assistance.

Conflict of interest The authors declare that they have no conflict of interest.

\section{References}

1. Vital Statistics Japan 2012. Cancer death (2011). Ministry of Health, Labor and Welfare. 2014. http://ganjoho.jp/data/public/ statistics/backnumber/2012/files/cancer_statistics_2012.pdf. Accessed 15 Aug 2014.

2. Warshaw AL, Fernández-del Castillo C. Pancreatic carcinoma. N Engl J Med. 1992;326:455-65.

3. Rosewicz S, Wiedenmann B. Pancreatic carcinoma. Lancet. 1997;349:485-9.

4. Furuta K, Watanabe H, Ikeda S. Differences between solid and duct-ectatic type of pancreatic ductal carcinoma. Cancer. 1992; 69:1327-33.

5. Egawa S, Toma H, Ohigashi H, et al. Japan Pancreatic Cancer Registry; 30th year anniversary. Pancreas. 2012;41:985-92.

6. Yamaguchi K, Okusaka T, Shimizu K, et al. EBM-based clinical guidelines for pancreatic cancer (2013) issued by the Japan Pancreatic Society: a synopsis. Jpn J Clin Oncol. 2014. doi:10. 1093/jjco/hyu127.

7. Uehara $\mathrm{H}$, Nakaizumi A, Ishikawa $\mathrm{O}$, et al. Development of ductal carcinoma of the pancreas during follow-up of branch duct intraductal papillary mucinous neoplasm of the pancreas. Gut. 2008;57:1561-5.

8. Hanada K, Amano H, Hino F, et al. Management strategies for branch duct intraductal papillary-mucinous neoplasms. Dig Endosc. 2006;18:S68-72.

9. Tada M, Kawase T, Arizumi M, et al. Pancreatic cancer in patients with pancreatic cystic lesions: a prospective study in 197 patients. Clin Gastroenterol Hepatol. 2006;4:1265-70.

10. Maguchi H, Tanno S, Mizuno N, et al. Natural history of branch duct intraductal papillary mucinous neoplasms of the pancreas. Pancreas. 2011;40:364-70.

11. Yamaguchi K, Kanemitsu S, Hatori T, et al. Pancreatic ductal adenocarcinoma derived from IPMN and pancreatic ductal adenocarcinoma concomitant with IPMN. Pancreas. 2011;40:571-80.

12. Klein AP. Identifying people at a high risk of developing pancreatic cancer. Nat Rev. 2013;13:66-74.

13. Canto MI, Goggins M, Yeo CJ, et al. Screening for pancreatic neoplasma in high-risk individuals: an EUS-based approach. Clin Gastroenterol Hepatol. 2004;2:606-21.
14. Hruban RH, Petersen GM, Goggins M, et al. Familiar pancreatic cancer. Ann Oncol. 1999;10(Suppl 4):69-73.

15. Kastrinos F, Fukushima N, Takaori K, et al. Risk of pancreatic cancer in families with Lynch syndrome. JAMA. 2009;302: $1790-5$.

16. Vesen HF, Gruis NA, Frants RR, et al. Risk of developing pancreatic cancer in families with familiar atypical multiple mole melanoma associated with a specific 19 deletion of p16 (p16 Leiden). Int J Cancer. 2000;87:809-11.

17. Poley JW, Kluijt I, Gouma DJ, et al. The yield of first-time endoscopic ultrasonography in screening individuals at a high risk of developing pancreatic cancer. Am J Gastroenteol. 2009; 104:2175-81.

18. Vasen HF, Wasser M, van Mil A, et al. Magnetic resonance imaging surveillance detects early-stage pancreatic cancer in carriers of a p16-Leiden mutation. Gastroenterology. 2011;140: $850-6$.

19. Riker A, Libutti SK, Bartlett DL. Advances in the early detection, diagnosis, and staging of pancreatic cancer. Surg Oncol. 1997;6:157-69.

20. Ballehaninna UK, Chamberlain RS. The clinical utility of serum CA19-9 in the diagnosis, prognosis and management of pancreatic adenocarcimona: an evidence based appraisal. J Gastrointest Oncol. 2012;3:105-19.

21. Nishiumi S, Shinohara M, Ikeda A, et al. Serum metabolomics as a novel diagnostic approach for pancreatic cancer. Metabolomics. 2010;6:518-28.

22. Kobayashi T, Nishiumi S, Ikeda A, et al. A novel serum metabolomics-based diagnostic approach to pancreatic cancer. Cancer Epidemiol Biomark Prev. 2013;22:571-9.

23. Yu J, Li A, Hong SM, et al. MicroRNA alterations of pancreatic intraepithelial neoplasias. Clin Cancer Res. 2012;18:981-92.

24. Tricoli JV, Jacobson JW. MicroRNA: potential for cancer detection, diagnosis, and prognosis. Cancer Res. 2007;67:4553-5.

25. Mori Y, Ohtsuka T, Kono $\mathrm{H}$, et al. A minimally invasive and simple screening test for detection of pancreatic ductal adenocarcinoma using biomarkers in duodenal juice. Pancreas. 2013;42:187-92.

26. Canto MI, Goggins M, Hruban RH, et al. Screening for early pancreatic neoplasia in high-risk individuals: a prospective controlled study. Clin Gastroenterol Hepatol. 2006;4:766-81.

27. Canto MI, Hruban RH, Fishman EK, et al. Frequent detection of pancreatic lesions in asymptomatic high-risk individuals. Gastroenterology. 2012;142:796-804.

28. Canto MI, Harinck F, Hruban RH, et al. International Cancer of the Pancreas Screening (CAPS) Consortium summit on the management of patients with increased risk for familiar pancreatic cancer. Gut. 2013;62:339-47.

29. Kamata K, Kitano M, Kudo S, et al. Value of EUS in early detection of pancreatic ductal adenocarcinomas in patients with intraductal papillary mucinous neoplasms. Endoscopy. 2014;46: $22-9$.

30. Tanaka S, Nakao M, Ioka T, et al. Slight dilatation of the main pancreatic duct and presence of pancreatic cysts as predictive signs of pancreatic cancer: a prospective study. Radiology. 2010; 254:965-72.

31. Hanada K, Iiboshi T, Hirano N, et al. Role of EUS for small pancreatic cancer less than $1 \mathrm{~cm}$. Tan Sui. 2009;30:343-8 (In Japanese).

32. Sakamoto H, Kitano M, Takeyama Y, et al. Early diagnosis of pancreatic cancer: approach to the diagnosis of very small pancreatic cancer with emphasis of US, MDCT and EUS. Tan Sui. 2009;30:335-41 (In Japanese).

33. Takeshita K, Kutomi K, Haruyama T, et al. Imaging of early pancreatic cancer on multidetector row helical computed tomography. Br J Radiol. 2010;83:823-30. 
34. Hijioka S, Ikari T, Kamei A, et al. CT and MRI findings with contrast enhancement of small pancreatic adenocarcinoma in the late phase. Hepatogastroenterology. 2007;54:389-92.

35. Serrano OK, Chaudhry MA, Leach SD, et al. The role of PET scanning in pancreatic cancer. Adv Surg. 2010;44:313-25.

36. Okano K, Kakinoki K, Akamoto S, et al. 18F-fluorodeoxyglucose positron emission tomography in the diagnosis of small pancreatic cancer. World J Gastroenterol. 2011;17:231-5.

37. Seo S, Doi R, Machimoto T, et al. Contribution of $18 \mathrm{~F}-$ fluorodeoxyglucose positron emission tomography to the diagnosis of early pancreatic carcinoma. J Hepatobiliary Pancreat Surg. 2008;15:634-9.

38. Matsumoto I, Shirakawa S, Shinzeki M, et al. 18F-fluorodeoxyglucose positron emission tomography does not aid in diagnosis of pancreatic ductal adenocarcinoma. Clin Gastroenterol Hepatol. 2013;11:712-8.

39. Maguchi $\mathrm{H}$. The roles of endoscopic ultrasonography in the diagnosis of pancreatic tumors. J Hepatobilbiliary Pancreat Surg. 2004;11:1-3.

40. Irisawa A, Sato A, Ikeda T, et al. Early diagnosis of small pancreatic cancer: role of endoscopic ultrasonography. Dig Endosc. 2009;21:S92-6.

41. Kimmey MB, Bronner MP, Byrd DR, et al. Screening and surveillance for hereditary pancreatic cancer. Gastrointest Endosc. 2002;56:S82-6.

42. Geenhalf W, Neoptolemos JP. Increasing survival rates of patients with pancreatic cancer by earlier identification. Nat Clin Pract Oncol. 2006;3:346-7.

43. Yasuda I, Iwashita T, Doi S, et al. Role of EUS in the early detection of small pancreatic cancer. Dig Endosc. 2011;23(Suppl 1):22-5

44. Uehara H, Ikezawa K, Kawada N, et al. Diagnostic accuracy of endoscopic ultrasound-guided fine needle aspiration for suspected pancreatic malignancy in relation to the size of lesions. J Gastroenterol Hepatol. 2011;26:1256-61.

45. Takagi T, Irisawa A, Sawaki A, et al. Effectiveness of EUS-FNA for the diagnosis of small pancreatic cancer less than $10 \mathrm{~mm}$. Tan Sui. 2009;30:361-7 (In Japanese).

46. Yokohata K, Shirahane H, Yonemasu T, et al. Focal ductal branch on magnetic resonance cholangiopancreatography: a hint for early diagnosis of pancreatic carcinoma. Scand J Gastroenterol. 2000;11:1229-32.

47. Hanada K, Iiboshi T, Yamao K, et al. Diagnostic strategies for the early diagnosis of pancreatic cancer. Gastroenterol Endosc. 2012;54:3773-82 (In Japanese with English abstract).
48. Seki M, Ninomiya E, Takano K, et al. Pancreatogram findings for carcinoma in situ (CIS) of the pancreas seen on endoscopic retrograde cholangiopancreatography and postoperative pancreatography of resected specimens: can CIS be diagnosed preoperatively? Pancreatology. 2008;8:142-52.

49. Ikeda S, Maeshiro K, Ryu S, et al. Diagnosis of small pancreatic cancer by endoscopic balloon-catheter spot pancreatography. Pancreas. 2009;38:e102-13.

50. Brune K, Abe T, Canto M, et al. Multifocal neoplastic precursor lesions associated with lobular atrophy of the pancreas having a strong family history of pancreatic cancer. Am J Surg Pathol. 2006;30:1067-76.

51. Iiboshi T, Hanada K, Fukuda T, et al. Value of cytodiagnosis using endoscopic nasopancreatic drainage for early diagnosis of pancreatic cancer. Pancreas. 2012;41:523-9.

52. Takaori K, Matsusue S, Fujikawa T, et al. Carcinoma in situ of the pancreas associated with localized fibrosis: a clue to early detection of neoplastic lesions arising from pancreatic ducts. Pancreas. 1998;17:102-5.

53. Nakaizumi A, Tatsuta $\mathrm{M}$, Uehara $\mathrm{H}$, et al. Cytologic examination of pure pancreatic juice in the diagnosis of pancreatic carcinoma. The endoscopic retrograde intraductal catheter aspiration cytologic technique. Cancer. 1992;70:2610-4.

54. Nakaizumi A, Tatsuta M, Uehara H, et al. Effectiveness of the cytologic examination of pure pancreatic juice in the diagnosis of early neoplasia of the pancreas. Cancer. 1995;76:750-7.

55. Mikata R, Ishihara $\mathrm{T}$, Tada $\mathrm{M}$, et al. Clinical usefulness of repeated pancreatic juice cytology via endoscopic naso-pancreatic drainage tube in patients with pancreatic cancer. J Gastroenterol. 2012;48:866-73.

56. Kimura K, Furukawa Y, Yamasaki S, et al. A study of the usefulness of pancreatic juice cytology obtained via an endoscopic nasal pancreatic drainage (ENPD) tube. Nihon Shokakibyo Gakkai Zasshi. 2011;108:928-36 (In Japanese with English abstract).

57. Hanada K, Iiboshi $\mathrm{T}$, Hirano $\mathrm{N}$, et al. A social program for diagnosis of the stage 0 pancreatic cancer in a rural doctor's association. Pancreas. 2012;41:1156.

58. Hanada K, Iiboshi $\mathrm{T}$. Recent advances in the early diagnosis of pancreatic cancer. Nihon Shokakibyo Gakkai Zasshi. 2013;110:2051-9 (In Japanese).

59. Fukasawa M, Yoda Y, Takayama I, et al. Regional cooperation aimed at the early detection of pancreatic cancer. Kan Tan Sui. 2013;66:277-84 (In Japanese). 